\title{
An Iterative Learning Control Technique for Point-to-Point Maneuvers Applied on an Overhead Crane
}

\author{
Khaled A. Alhazza, ${ }^{1}$ Abdullah M. Hasan, ${ }^{1}$ Khaled A. Alghanim, ${ }^{1}$ and Ziyad N. Masoud ${ }^{2}$ \\ ${ }^{1}$ Department of Mechanical Engineering, Kuwait University, P.O. Box 5969, Safat, 13060 Kuwait City, Kuwait \\ ${ }^{2}$ Department of Mechatronics Engineering, The German-Jordanian University, Amman 11180, Jordan \\ Correspondence should be addressed to Khaled A. Alhazza; kalhazza@vt.edu
}

Received 14 March 2014; Accepted 28 April 2014; Published 12 May 2014

Academic Editor: Mohammad Elahinia

Copyright (c) 2014 Khaled A. Alhazza et al. This is an open access article distributed under the Creative Commons Attribution License, which permits unrestricted use, distribution, and reproduction in any medium, provided the original work is properly cited.

\begin{abstract}
An iterative learning control (ILC) strategy is proposed, and implemented on simple pendulum and double pendulum models of an overhead crane undergoing simultaneous traveling and hoisting maneuvers. The approach is based on generating shaped commands using the full nonlinear equations of motion combined with the iterative learning control, to use as acceleration commands to the jib of the crane. These acceleration commands are tuned to eliminate residual oscillations in rest-to-rest maneuvers. The performance of the proposed strategy is tested using an experimental scaled model of an overhead crane with hoisting. The shaped command is derived analytically and validated experimentally. Results obtained showed that the proposed ILC control strategy is capable of eliminating travel and residual oscillations in simple and double pendulum models with hoisting. It is also shown, in all cases, that the proposed approach has a low sensitivity to the initial cable lengths.
\end{abstract}

\section{Introduction}

One of the major problems in industries that involve crane operations is payload oscillations in point-to-point crane maneuvers. Many control techniques have been developed in the past few decades to reduce these residual oscillations. Point-to-point maneuvers of cranes pose a major challenge to crane controller designers. A large bulk of research have been devoted to dynamics and control of cranes [1]. The main objective of such research is to eliminate the travel and residual oscillations and/or reduce maneuvers time.

Massive payloads are generally transferred using cranes. Inefficient crane operations can cause substantial production delays, devastating property damage, and even loss of lives. To maintain fast and safe crane operations, many feedback and feedforward control techniques were proposed. Researchers have successfully used feedback for oscillation control of cranes [2-7]. Although feedback controllers can effectively reduce residual oscillations, these controllers may require installation of additional hardware on the crane or in some cases alteration to the existing system. These changes may be costly or impractical.
To overcome such disadvantages of feedback controllers, open-loop techniques are becoming more popular especially in point-to-point maneuvers. Input-shaping crane control strategy is considered one of the most commonly used openloop techniques and it can be defined as a method of reducing residual oscillations by convolving a sequence of impulses with a baseline reference command [8-10]. If the baseline command is constant, then the produced command is an input-shaped step function.

Several input-shaping techniques were derived in the past four decades. Early step shapers were designed to eliminate the residual oscillations of crane payloads at the modeled natural frequency and damping ratio. These shapers are known as zero vibration (ZV) input shaper. Many command shapers have been developed later on to increase the robustness of the ZV shaper. Vaughan et al. [10] compromised between rapidity of motion and shaper robustness for several inputshaping methods. They showed that robust shapers typically have longer durations that slow the system response. Using simulation and experiments, Terashima et al. [11] developed a three-dimensional open-loop control strategy for sway-free, point-to-point maneuvers of a rotary crane. They showed 
that the proposed control method is effective in eliminating residual oscillations and centrifugal force influence. Starr [12] used a symmetric double-step acceleration profile to transport a suspended object with minimal oscillations. A linear approximation of the oscillation period was used to calculate the switching times and to generate an analytical expression for the acceleration profile. This work was later extended by employing a nonlinear approximation of the payload frequency to generate single-step and double-step symmetric acceleration profiles [13].

Kress et al. [14] proved analytically that input shaping is equivalent to a notch filter applied to a general input signal and centered around the natural frequency of the payload oscillations. They applied a second-order robust notch filter to shape the acceleration input of a gantry crane. Numerical simulation and experimental verification of this strategy implemented on an actual bidirectional crane, moving at arbitrary step acceleration and changing cable length at a slow constant speed, showed that the strategy was able to efficiently suppress residual payload oscillation. Their work was later extended by developing an inputshaping notch filter to reduce payload oscillation of rotary cranes excited by operator commands. It was reported that, in general, there was no guarantee that applying such filter to the operator's speed commands would result in excitation terms having the desired frequency content and that it only works for low speed and acceleration commands [15]. Parker et al. [16] experimentally verified their numerical simulation results.

Other types of command shapers were used in the literature. A new single stage variable acceleration profile was introduced by Alhazza and Masoud [17]. They successfully eliminated travel and residual payload oscillations of an overhead crane. Their approach resulted in an acceleration profile which was smoother compared to the double-step acceleration profile. Their work was later extended to include the effect of damping [18]. Numerical simulations and experiments proved that the proposed profiles were capable of eliminating residual oscillations for different damping ratios [19].

A significant part of the research on crane control was focused on simple pendulum models of a crane with a constant cable length, to avoid the complexity of analyses that include hoisting. It is well known that hoisting introduces time varying parameters in the equations of motion. These parameters make solving such equations very challenging. Following this line of research, many researchers introduced new shapers and techniques. Singhose et al. [20] studied four different input-shaping controllers considering the effect of hoisting. In their work, they reported that the best inputshaper produced a reduction of $73 \%$ in transient oscillations over the time-optimal rigid-body commands. They also showed that input-shaping technique using constant length can reduce residual vibrations to acceptable levels without eliminating them. Masoud and Daqaq [21] successfully extended the double-step shaped-commands to accommodate large hoisting maneuvers. Performance was validated on a scaled experimental model of a container crane performing large simultaneous hoisting and traversing maneuvers.
New line of research has emerged recently on command shaping for double pendulum models of cranes. The interest in such research is due to the fact that the mass of the hook used, in heavy lift cranes, can be so large that the payload and the hoisting mechanism may exhibit a double pendulum behavior [22, 23]. In such application, designing a control system using a simple pendulum model suffers a major drawback due to the uncontrolled second mode oscillations. Such control systems may even induce larger second mode oscillation amplitude.

In multidegrees of freedom systems, command shapers designed for the first mode may eliminate higher modes of oscillations provided that their frequencies are odd integer multiples of the first mode frequency. Using this fact, Masoud et al. [24] and Masoud and Alhazza [25] used modelbased feedback to develop command shapers for a double pendulum model of an overhead crane.

A rest-to-rest maneuver is a repeated process that executes the same task with the same initial conditions. This fact made the iterative learning control (ILC) technique a perfect candidate for such application. There are many learning control techniques, such as adaptive control, neural network, and repetitive control (RC). Iterative learning control method is a cyclical process that modifies the input commands at each cycle or repetition. Research using this technique faces many challenges, especially because of jerks in the command profiles and slow system rise time. Wang et al. [26] reviewed and compared three different control methods: iterative learning control (ILC), repetitive control (RC), and run-to-run control (R2R). In their work, they approximately categorized 400 papers related to learning-type control. In addition, a flowchart based on the unique features of the different methods is presented as a guideline for choosing an appropriate learning-type control for different problems. Scardua et al. [27] studied the use of reinforcement learning (RL) for developing a time-optimal antiswing control of a ship unloader. They used double-step functions to optimize the output. In real world, there are many industrial systems that perform repeated processes. ILC has been used successfully in many applications [28-42].

In the present work, iterative learning control technique is proposed and implemented experimentally on a model of an overhead crane to reduce residual oscillations of simple and double pendulum systems with hoisting. The approach is based on generating a discrete time shaped acceleration command profile using the full nonlinear equations of motion, complemented with iterative learning control technique. The number of discrete time points is chosen based on the level of accuracy required or in accordance with the actual experimental crane minimum time steps. The proposed approach modifies input commands at each repetition of a cyclical process. As the learning process makes more progress in identifying the system's output, the predicted next repetition output comes closer to the actual output, and, consequently, the learning method yields improved results. The controller parameters, such as time, maximum acceleration, maximum velocity, and acceleration jumps or jerks, are tuned to eliminate residual oscillations in rest-to-rest maneuvers. The controller performance is 


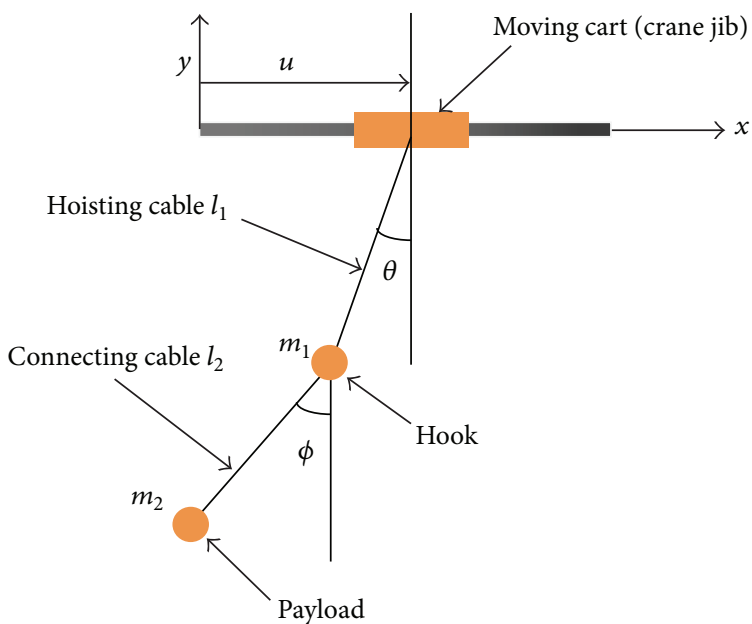

FIGURE 1: Overhead crane with a massive hook model.

determined analytically and validated experimentally on a scaled model of an overhead crane. Results obtained showed that the proposed control technique is capable of eliminating residual oscillations in both simple and double pendulum cases.

\section{Mathematical Model}

Most of the research on crane control simplifies the crane dynamics as a simple pendulum system. Although this assumption is valid for some cases and can result in significant performance improvement, large payloads or massive hooks may give rise to significant second mode oscillations. In such cases, single mode controller would produce suboptimal performance. To solve this problem, double pendulum models are used to represent cranes with large payloads.

In this work, an overhead crane with a large hook is modeled as a double pendulum. The length of the hoisting cable, $l_{1}$, is considered variable; see Figure 1 . The length $l_{2}$ of the cable connecting the payload to the hook of the crane is assumed to be constant. The position vectors to the hook, $m_{1}$, and the payload of the crane, $m_{2}$, are

$$
\begin{array}{r}
\mathbf{p}_{1}=\left[u-l_{1} \sin (\theta),-l_{1} \cos (\theta)\right], \\
\mathbf{p}_{2}=\left[u-l_{1} \sin (\theta)-l_{2} \sin (\phi),\right. \\
\left.-l_{1} \cos (\theta)-l_{2} \cos (\phi)\right] .
\end{array}
$$

Using (1), the kinetic and potential energies are

$$
\begin{aligned}
T & =\frac{1}{2} m_{1} \dot{\mathbf{p}}_{1} \cdot \dot{\mathbf{p}}_{1}+\frac{1}{2} m_{2} \dot{\mathbf{p}}_{2} \cdot \dot{\mathbf{p}}_{2}, \\
V & =m_{1} g p_{1 y}+m_{2} g p_{2 y} .
\end{aligned}
$$

Applying Lagrange's formulation, the nonlinear equations of motion of the double pendulum model in terms of the generalized coordinates $\theta$ and $\phi$ are

$$
\begin{aligned}
& \left(m_{1}+m_{2}\right)\left[l_{1}^{2} \ddot{\theta}+l_{1} g \sin (\theta)+2 l_{1} \dot{l}_{1} \dot{\theta}\right] \\
& +m_{2} l_{1} l_{2}\left[\ddot{\phi} \cos (\theta-\phi)+\dot{\phi}^{2} \sin (\theta-\phi)\right] \\
& \quad=\left(m_{1}+m_{2}\right) l_{1} \cos (\theta) \ddot{u}, \\
& m_{2} l_{2}^{2} \ddot{\phi}+m_{2} l_{2} \ddot{l}_{1} \sin (\theta-\phi) \\
& -m_{2} l_{2} l_{1}\left[\dot{\theta}^{2} \sin (\theta-\phi)-\ddot{\theta} \cos (\theta-\phi)\right] \\
& +2 m_{2} l_{2} \dot{l}_{1} \dot{\theta} \cos (\theta-\phi)+m_{2} g l_{2} \sin (\phi) \\
& =m_{2} l_{2} \cos (\phi) \ddot{u} .
\end{aligned}
$$

\section{Learning Control}

Learning control method is a cyclical process that, in general, modifies the input commands in repeated cycles. Each cycle starts with, typically, the same initial conditions. Identifying effect of the system's input on the system's output directly, without taking into consideration the dynamics of the system, is the key behind learning control process. The following derivation clarifies the concept of learning control technique. Consider a cyclic system of piecewise step inputs at fixed time steps; the system output at any repetition can be expressed as

$$
Y_{i}=B_{i} U_{i}+\mathrm{er}_{i}
$$

where

$$
\begin{aligned}
& Y_{i}=\left[\begin{array}{llll}
y_{i}(\Delta t) & y_{i}(2 \Delta t) & y_{i}(3 \Delta t) & \cdots
\end{array}\right. \\
& \left.y_{i}((p-1) \Delta t) \quad y_{i}(p \Delta t)\right]^{T} \\
& U_{i}=\left[\begin{array}{llll}
u_{i}(0) & u_{i}(\Delta t) & u_{i}(2 \Delta t) & \cdots
\end{array}\right. \\
& \left.u_{i}((p-2) \Delta t) u_{i}((p-1) \Delta t)\right]^{T} .
\end{aligned}
$$

$Y_{i}$ is the system output vector of length $p, B_{i}$ is the $p \times$ $p$ system parameter matrix, $U_{i}$ is the system input vector of length $p$, and $\mathrm{er}_{i}$ is the estimated error vector of length $p$, with all being at the $i$ th repetition.

The system parameter matrix $B$ can be initiated arbitrarily; however, the initial estimation of this matrix has a considerable effect on the progression of the identification process. Since the exact parameter matrix is unpredictable, it is suggested to start with the identity matrix as an initial estimation of $B$. By assuming a very small error $\mathrm{er}_{i}$ in (4), the next repetition output is expressed as

$$
Y_{i+1}=B_{i+1} U_{i+1} \text {. }
$$

Since $B_{i+1}$ cannot be predicted without knowing $Y_{i+1}$, therefore, assuming a small change in the matrix $B$ between each two consecutive iterations, $B_{i}$ will be used instead of $B_{i+1}$. Therefore, (6) can be written as

$$
Y_{i+1} \approx B_{i} U_{i+1}=B_{i}\left(U_{i}+\delta U_{i+1}\right)=Y_{i}+B_{i} \delta U_{i+1}
$$


and the difference between the actual output and desired output is given by

$$
e_{i+1}=Y^{*}-Y_{i+1}=Y^{*}-Y_{i}-B_{i} \delta U_{i+1},
$$

where $e_{i}$ is the variation between the actual and desired output at the $i$ th repetition and $Y^{*}$ is the desired output. As more information pertaining to the system is acquired, the estimated output that comes from matrices calculations becomes closer to the system's actual output. In sequence, the variation and error term decrease and $Y_{i+1}$ becomes closer to $Y^{*}$.

For explaining the optimizing process, consider a fitness function $J$ that needs to be minimized as follows:

$$
J=\frac{1}{2} e_{i+1}^{T} Q e_{i+1}+\frac{1}{2} \delta U_{i+1}^{T} S \delta U_{i+1},
$$

where $Q$ and $S$ are weighing matrices. In fact, matrix $Q$ works to put more effort in minimizing the variation, while $S$ tries to minimize the input differences among repetitions.

Substituting $Y_{i+1}$ from (7) into (9), the resulted equation is differentiated with respect to $\delta U_{i+1}$, which gives

$$
\frac{\partial J}{\partial \delta U_{i+1}}=-B_{i}^{T} Q\left(Y^{*}-Y_{i}\right)+\delta U_{i+1}^{T} B_{i}^{T} Q B_{i}+\delta U_{i+1}^{T} S .
$$

To solve for the optimal input, we set (10) to zero and solve for $\delta U_{i+1}$, which leads to

$$
\delta U_{i+1}=\left(B_{i}^{T} Q B_{i}+S\right)^{-1} B_{i}^{T} Q\left(Y^{*}-Y_{i}\right),
$$

where

$$
U_{i+1}=U_{i}+\delta U_{i+1} .
$$

In order to reduce jumps between input points or jerks, the differences between input values at the same repetition should be minimized; therefore, a smoothing matrix $K$ is introduced as

$$
K= \begin{cases}1, & i=j, \\ -1, & j=i+1, \\ 0, & i \neq j, \quad j \neq i+1\end{cases}
$$

and the term

$$
\frac{1}{2}\left(K \delta_{i}\right)^{T} W\left(K \delta_{i}\right)
$$

is added to (9). In result, (11) becomes

$$
\begin{aligned}
\delta U_{i+1}= & \left(B_{i}^{T} Q B_{i}+S+K^{T} W K\right)^{-1} \\
& \times\left[B_{i}^{T} Q\left(Y^{*}-Y_{i}\right)-K^{T} W K U_{i}\right],
\end{aligned}
$$

where $W$ is weighing identity matrix of smoothing term. Matrix $W$ works to put more effort in minimizing input differences in the same repetition.

To keep the system parameter matrix applicable in estimating the next repetition output and to keep the learning process within its range, reducing changes among repetitions in the system's input is important. If the system's input varies widely in two consecutive repetitions, the learning and identifying processes become unfeasible. So, it is vital to maintain the differences in the input small enough.

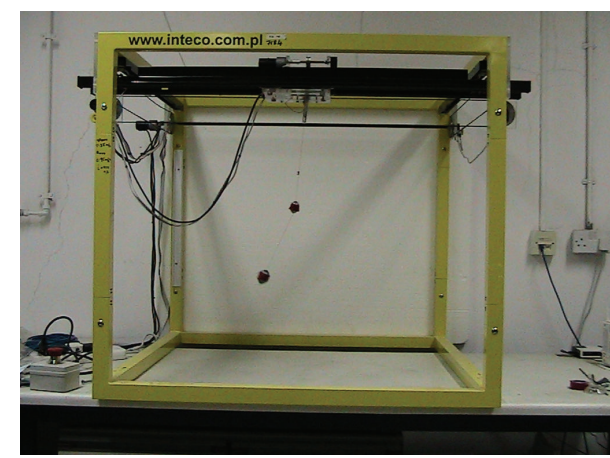

FIGURE 2: Experimental model.

\section{Numerical and Experimental Validation}

To verify the performance of the proposed method, four test cases are presented. The first two cases are of a simple pendulum with a constant cable length and a simple pendulum with hoisting. The other two cases are of a double pendulum with a constant cable length and a double pendulum with a variable hoisting cable length.

A MATLAB code is used to calculate the optimized acceleration of the jib of the crane. This acceleration is used to numerically solve the equations of motion; see (3). Results are then compared with results obtained using an experimental model of the overhead crane (see Figure 2).

The experimental model of the overhead crane is a $3 \mathrm{D}$ crane made by INTECO. The model is located in the Advanced Vibration Lab at Kuwait University. The motions of the jib and the bridge of the crane are controlled by two identical DC motors. Five optical encoders are used to measure the state variables of the crane; two encoders measure the jib and bridge motions, two encoders measure the oscillation angles of the hoisting cable, and one encoder measures the length of the hoisting cable. The resolution of the encoders is 1024 pulses per rotation. The interface between a PC running MATLAB's real-time Simulink environment and the crane setup is achieved using an RT-DAC/PCI multipurpose digital I/O board. The jib of the crane has a maximum speed of $0.3 \mathrm{~m} / \mathrm{s}$ and a maximum acceleration of $0.9 \mathrm{~m} / \mathrm{s}^{2}$. The crane has a $0.6 \mathrm{~m}$ usable jib and bridge tracks. Light weight cables are used with two masses representing the crane hook and payload.

In the following tests, the bridge of the crane is fixed and only planar motion is considered. The incremental encoder installed on the jib of the crane, which measures the oscillation angle, $\theta$, of the hoisting cable, is used for monitoring purposes only. It is important here to emphasize that the proposed control technique is an open-loop technique.

4.1. Simple Pendulum Crane with a Constant Cable Length. The length of the hoisting cable length is set to $0.4 \mathrm{~m}$. The mass of the payload is $0.21 \mathrm{~kg}$. Using these parameters, the optimized acceleration profile is generated as shown in Figure 3(a). The velocity and the displacement of the jib are also shown in Figure 3(a). The maximum velocity of the jib 


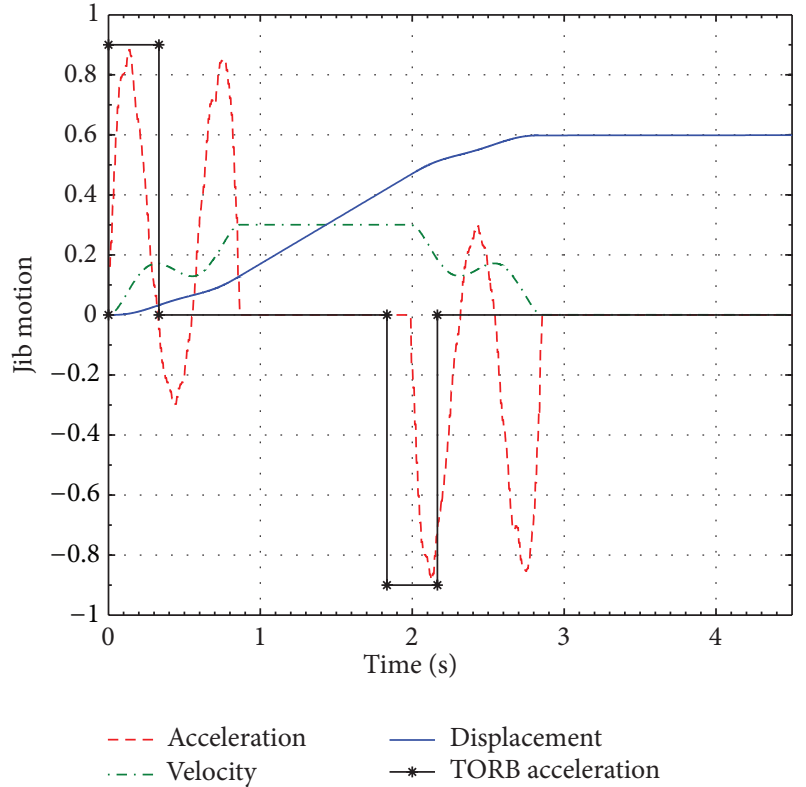

(a) TORB and ILC acceleration profiles

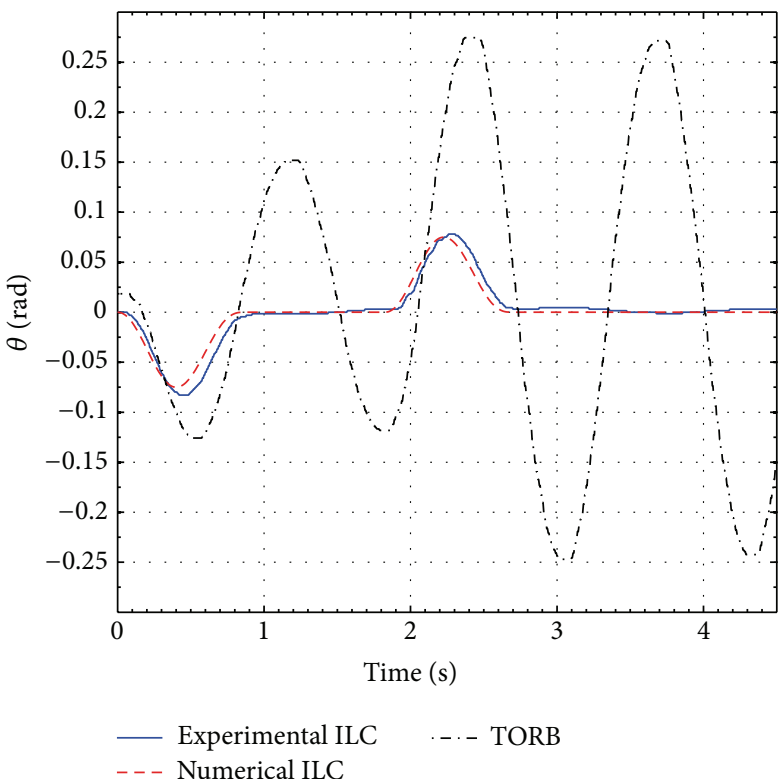

(b) Oscillation angle of the hoisting cable

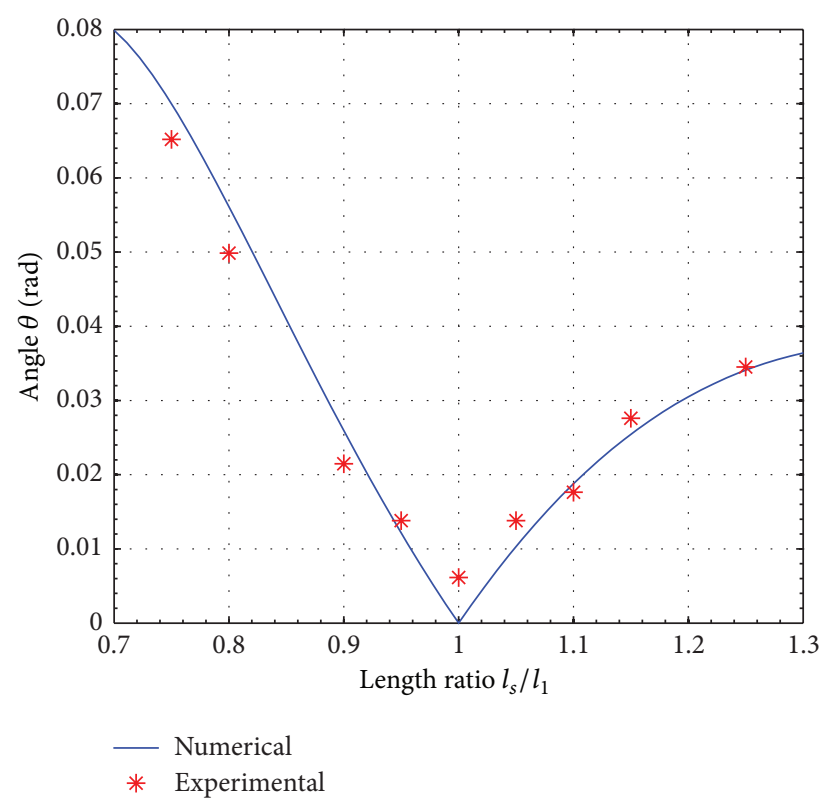

(c) Residual oscillation sensitivity

FIGURE 3: Simulations and experiments on a simple pendulum for $m=0.21 \mathrm{~kg}$ payload and $l=0.4 \mathrm{~m}$ hoisting cable.

and a displacement of $0.55 \mathrm{~m}$ are used as constraints for the ILC. It is clear that the proposed ILC technique is capable of utilizing the full capability of the crane.

Without a reference to the payload oscillations, the fastest maneuver is achieved using the time-optimal rigid-body (TORB) acceleration profile. Based on the jib maximum velocity and acceleration, the time needed to complete a TORB maneuver is 2.2 seconds.

Experimental and simulated oscillation angle of the hoisting cable, as shown in Figure 3(b), demonstrate the effectiveness of the ILC technique in eliminating residual oscillations. A small discrepancy between the experimental and simulated results is observed, which is attributed to many unmodeled nonlinearities, such as friction and mechanical backlash. Further examination of Figure 3(b) shows that the maximum transient oscillation angle using the ILC profile is $0.08 \mathrm{rad}$ while it rises to $0.15 \mathrm{rad}$ using the TORB profile. Furthermore, residual oscillations are reduced by approximately $99 \%$. The complete maneuver time of the ILC is 2.9 seconds. Despite being longer than the TORB maneuver time, the extra time is a small price to pay for the $99 \%$ reduction in the residual oscillation. The sensitivity of ILC to modeling 


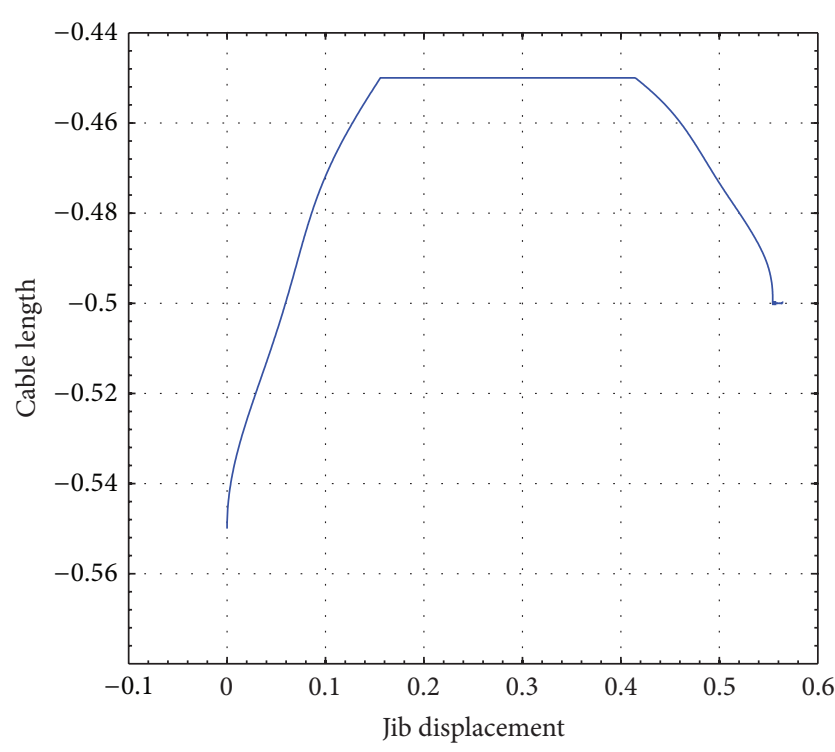

(a) Maneuver profile

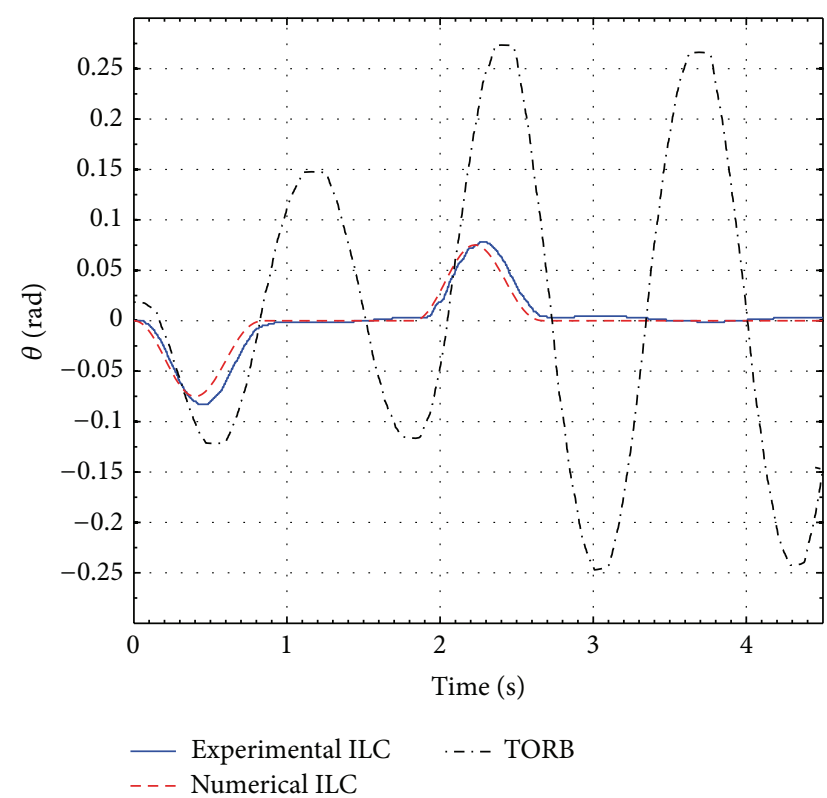

(c) Oscillation angle of the hoisting cable

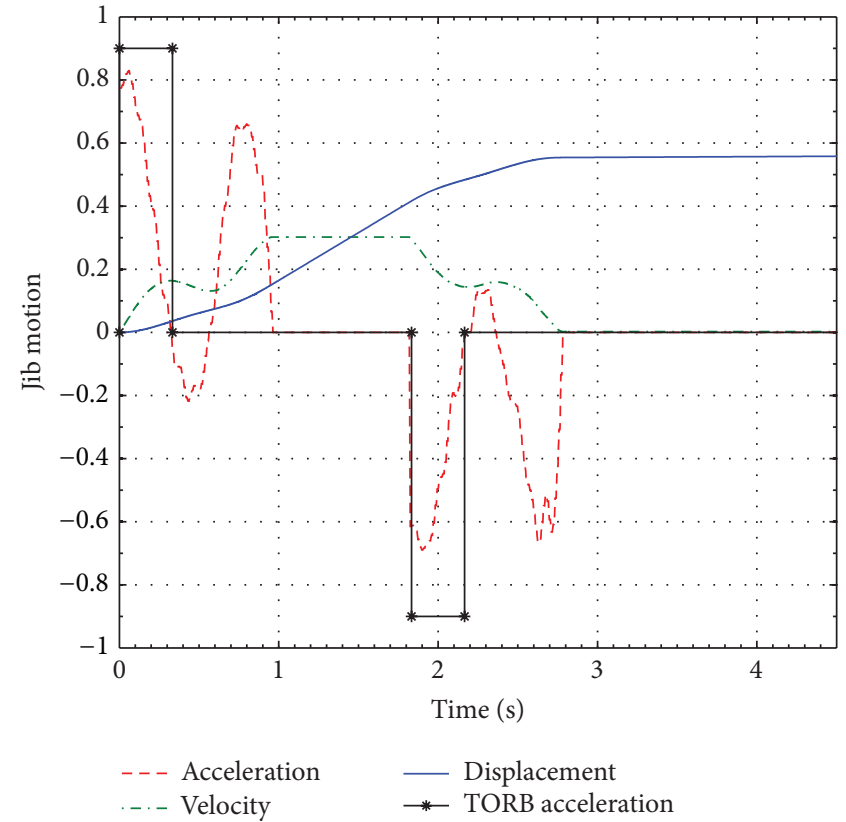

(b) ILC and TORB acceleration profiles

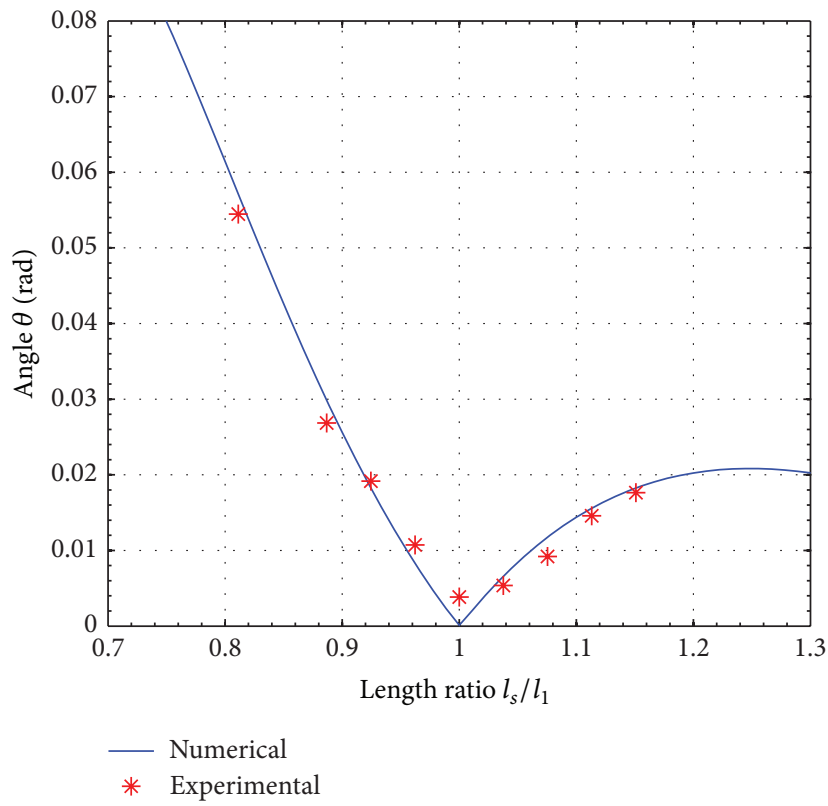

(d) Residual oscillation sensitivity

FIgURE 4: Simulations and experiments on a simple pendulum for $m=0.21 \mathrm{~kg}$ and variable hoisting cable length.

error in the hoisting cable length, and hence in the design frequency, is shown in Figure 3(c). Further examination of Figure 3(c) shows that $10 \%$ error in initial cable length results in approximately a 0.02 -radian final residual angle. With this large initial error of $10 \%$, the performance of the proposed technique sustains a reduction of almost $92 \%$ compared to the TORB.

4.2. Simple Pendulum Crane with Hoisting. In this test, the complexity of the task increases due to the effect of hoisting on the system dynamics. Simultaneous hoisting and jib motion are performed. Hoisting is assumed to be linear as

$$
l=l_{0}+R t,
$$

where $R$ is the hoisting speed. The length of the hoisting cable is changed from $0.55 \mathrm{~m}$ to $0.45 \mathrm{~m}$ during the acceleration phase and then increased from $0.45 \mathrm{~m}$ to $0.5 \mathrm{~m}$ in the deceleration phase; see Figure 4(a). The acceleration profiles in Figure 4(b) show a small time penalty on the ILC profile compared to the TORB complete maneuver time. 


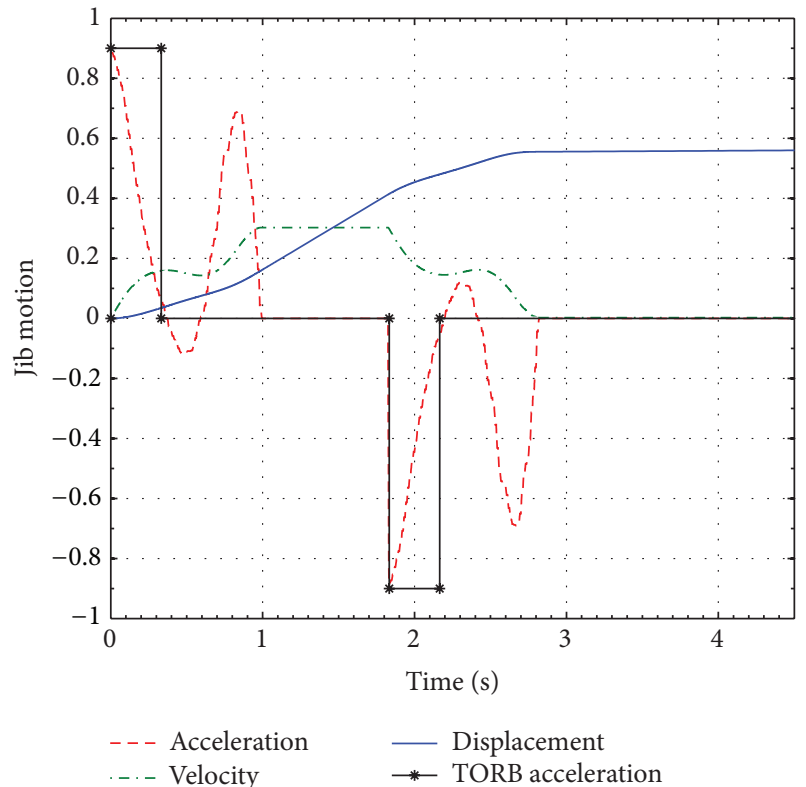

(a) ILC and TORB acceleration profiles

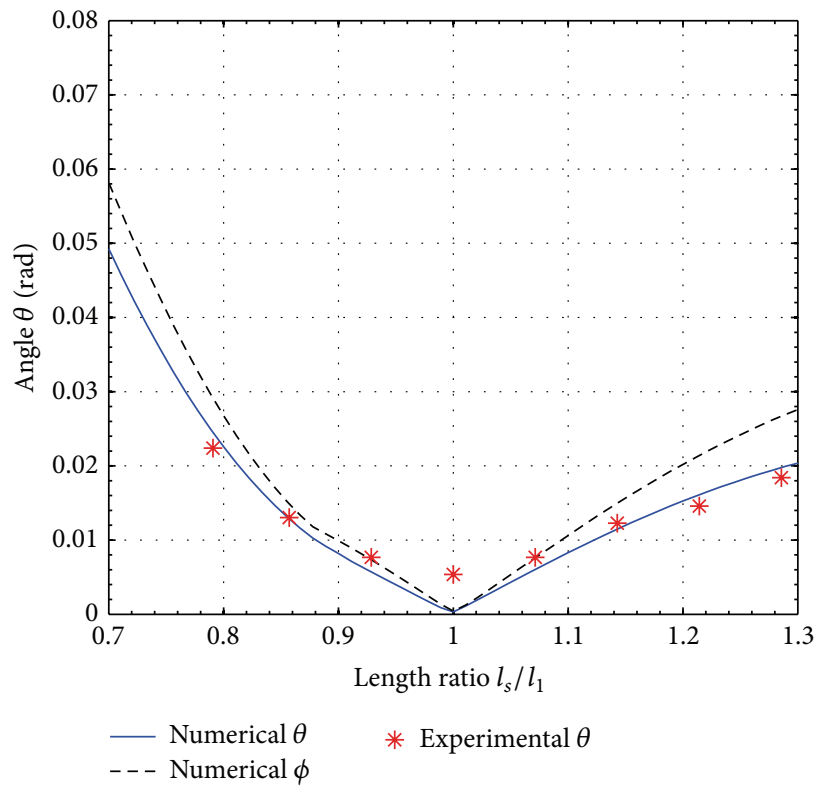

(c) Sensitivity of residual oscillations of $l_{1}$

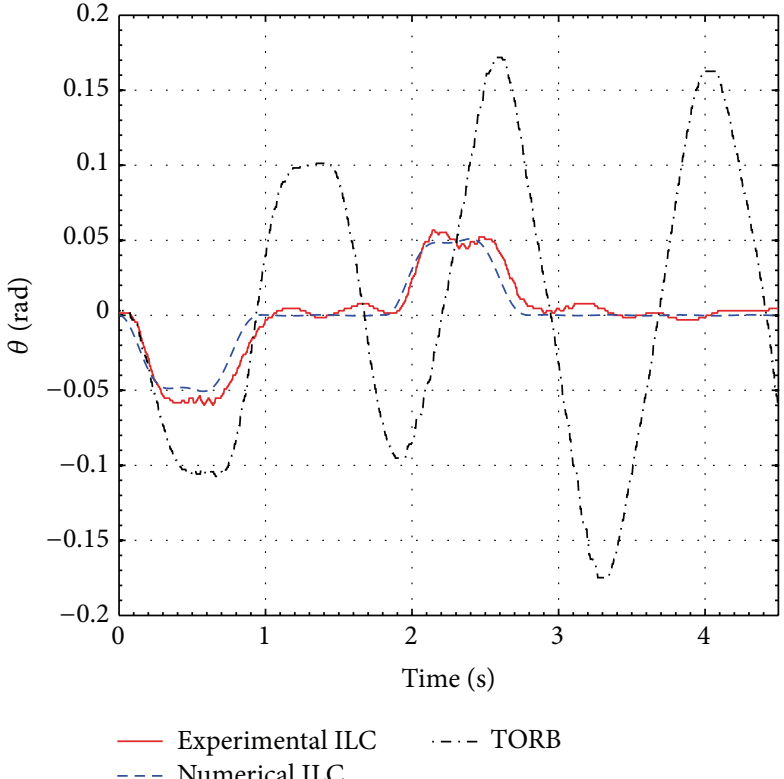

(b) Oscillation angle of the hoisting cable

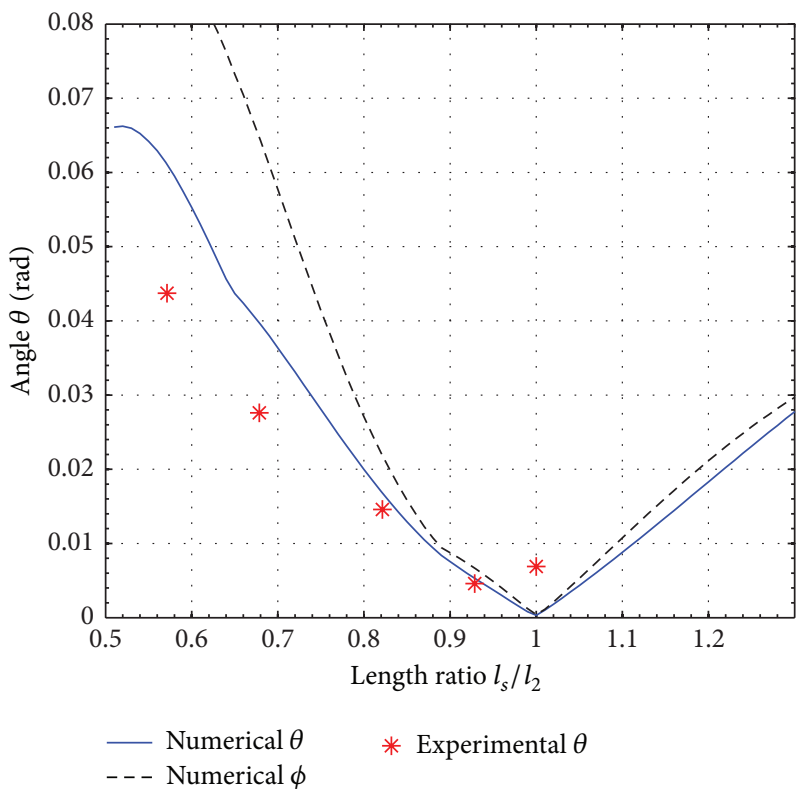

(d) Sensitivity of residual oscillations of $l_{2}$

FIGURE 5: Simulations and experiments on a double pendulum with no hoisting.

The change in the hoisting cable length used is limited by the $0.12 \mathrm{~m} / \mathrm{s}$ maximum hoisting speed of the experimental crane. Significant reduction in residual oscillations is achieved; see Figure 4(c). The sensitivity of ILC to modeling errors is shown in Figure 4(d). Comparing Figure 4(d) with Figure 3(c) shows that underestimating the initial cable length results in an increase in the final residual vibration while overestimating the initial length results in a decrease in the final residual angle. It is worth noting that the sensitivity curves are a function of the initial length, velocity, hoisting speed, and acceleration. Changing any of these parameters results in a small change in the sensitivity curve but keeps the general shape.

4.3. Double Pendulum Crane with a Constant Hoisting Cable Length. In this test, a double pendulum is attached to the jib of the crane with an upper mass of $0.105 \mathrm{~kg}$ representing the hook of the crane and a lower mass of $0.210 \mathrm{~kg}$ representing the crane payload. The length of the hoisting cable is set to $l_{1}=$ $0.40 \mathrm{~m}$ while the length of the connecting length is arbitrarily set to $l_{2}=0.17 \mathrm{~m}$ (see Figure 1 ). 


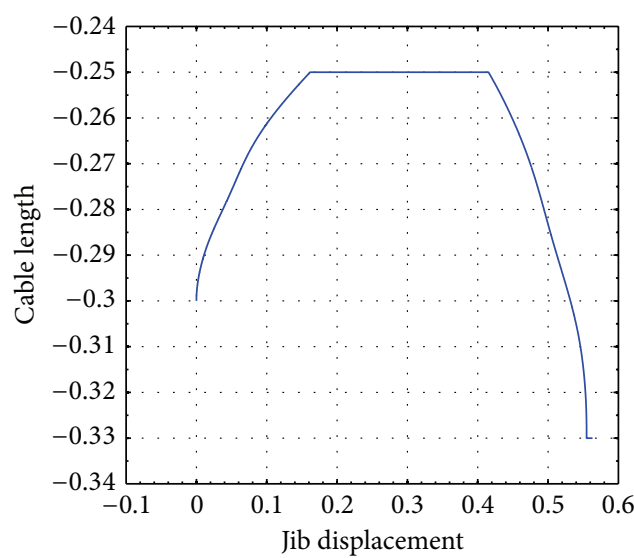

(a) Maneuver profile

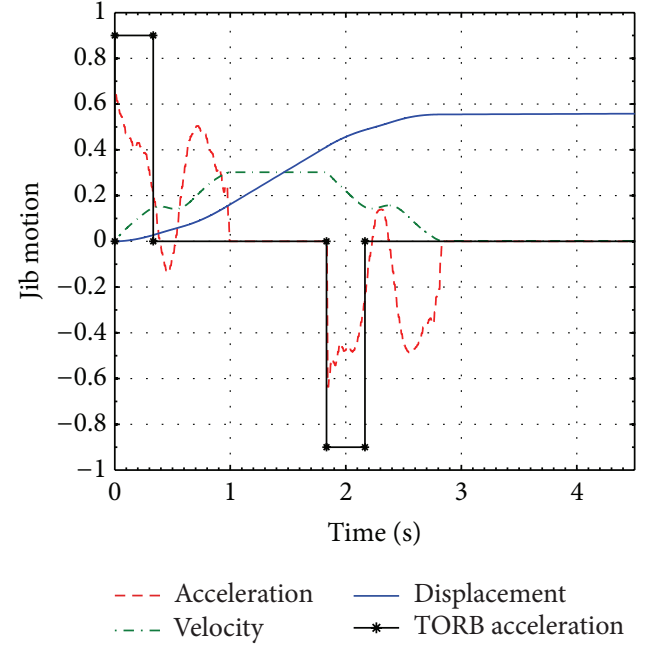

(b) TORB and ILC acceleration commands

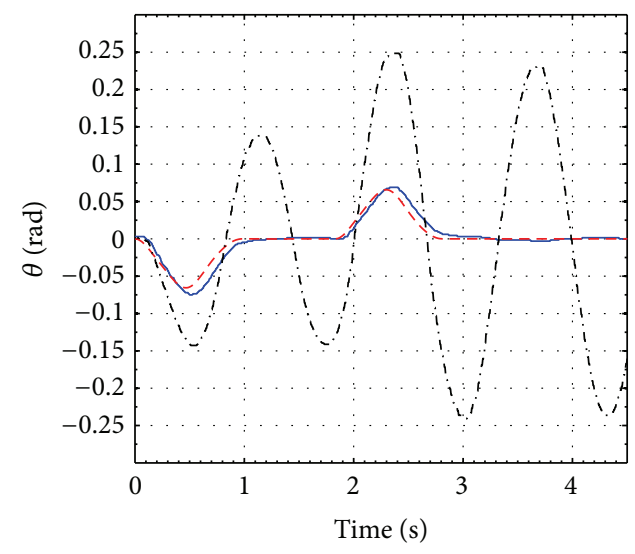

- Experimental ILC

...- TORB

- - Numerical ILC

(c) Oscillation angle of the hoisting cable

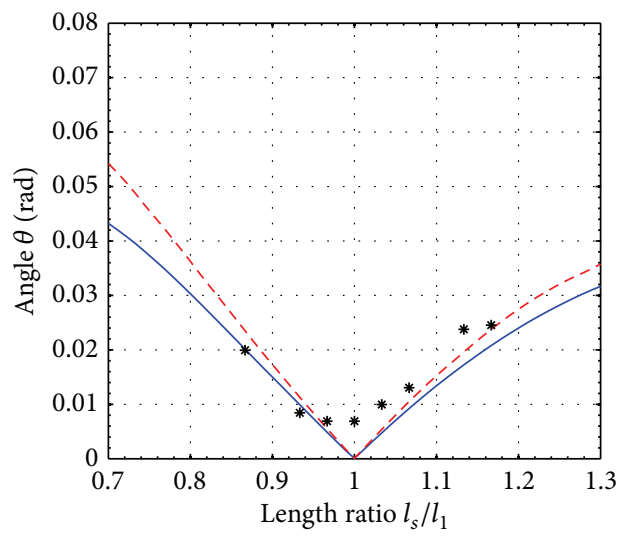

- Numerical $\theta$
-- Numerical $\phi$

(d) Sensitivity of residual oscillations of $l_{1}$

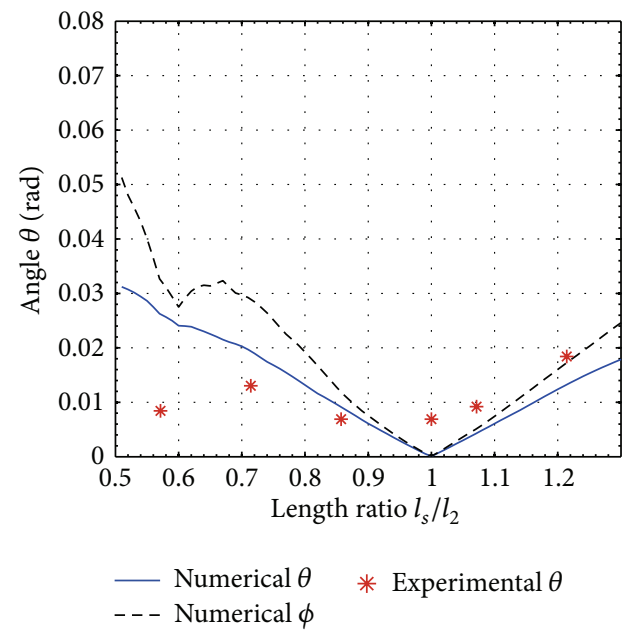

(e) Sensitivity of residual oscillations of $l_{2}$

FIGURE 6: Simulations and experiments on a double pendulum with hoisting. 
In the double pendulum test cases, only the oscillation angle of the hoisting cable is measured. Figure 5(a) shows that the ILC profile satisfies the maximum velocity and displacement constraints.

Figure 5(b) shows some discrepancy between simulated and measured oscillation angle due to modeling uncertainties and neglected nonlinearities. Due to this discrepancy, measured residual oscillations were reduced by $98 \%$ compared to the complete elimination of these oscillations in simulated results. A small time penalty of approximately 0.6 seconds was the price of that $98 \%$ oscillation reduction.

The sensitivity of residual oscillations in the angles of the hoisting and connecting cables is shown in Figures 5(c) and $5(d)$, respectively. Figure 5(c) shows that a $10 \%$ error in the initial length results in a final residual vibration of only 0.01 radian and $20 \%$ error results in an angle less than 0.02 radian. The same observation is noticed in Figure 5(d). An interesting observation is obtained by further inspection of these figures, which shows that the ILC is less sensitive to modeling errors in the case of a double pendulum model compared to the simple pendulum model case. Again, changing any of the system parameters results in a small quantitative change in the performance of the controller.

4.4. Double Pendulum Crane with Hoisting. In this test, the length of the hoisting cable is changed linearly as in (16). This change in length is very challenging since both frequencies of the double pendulum change during maneuver. In this test, hoisting takes place during the acceleration and deceleration phases only.

The initial cable length was set to $0.3 \mathrm{~m}$. The cruising cable length was $0.25 \mathrm{~m}$. The final hoisting cable length at the end of the maneuver was $0.33 \mathrm{~m}$; see Figure 6(a). ILC and TORB inputs are shown in Figure 6(b). It is clear that, even with such a complicated case, ILC successfully satisfies system constraints in terms of maximum velocity and displacement. Despite the complexity of the test, Figure 6(c) shows excellent performance of the ILC both simulated and measured, and the residual vibrations are reduced by almost $99 \%$.

Sensitivity curves for both oscillation angles of the hoisting and the connecting cables are shown in Figures 6(d) and 6(e), respectively. Figure 6(d) shows that a 10\% change in the initial length estimation results in a small residual angle of 0.015 radian. This result showed that, unlike the simple pendulum, the effect of hoisting increased the sensitivity of the controller performance. On the other hand, Figure 6(e) shows that a $10 \%$ error in the initial estimation results in only a 0.005 -radian residual angle. This result showed that the hoisting reduces the sensitivity of the system to errors in the initial mass estimation.

It is worth noting that the proposed technique successfully handled numerically all the cases examined even with high hoisting speed, larger acceleration, small initial length, different number of points, and so forth. All parameters in the demonstrated cases examined, such as the number of points, maximum acceleration, distance, maximum speed, and hoisting speed, are chosen to be within the experimental constrains.

\section{Conclusions}

In the present work, an iterative learning control technique is proposed to generate acceleration profiles of overhead crane maneuvers involving hoisting. The proposed ILC technique is most useful for applications where repeated automated maneuvers are involved. Simulations and experiments demonstrate the effectiveness of the proposed ILC technique in eliminating residual oscillations in simple pendulum and double pendulum models of overhead cranes. While completely eliminated in simulations, experiments on restto-rest maneuvers using ILC result in a reduction of residual oscillations of approximately 98\%. The ILC technique is shown to handle maneuvers involving hoisting as effectively as cases with no hoisting with small calculating efforts. The ILC demonstrated significant robustness and reduced sensitivity to modeling errors and uncertainties.

\section{Conflict of Interests}

The authors declare that there is no conflict of interests regarding the publication of this paper.

\section{References}

[1] E. M. Abdel-Rahman, A. H. Nayfeh, and Z. N. Masoud, "Dynamics and control of cranes: a review," Journal of Vibration and Control, vol. 9, no. 7, pp. 863-908, 2003.

[2] Z. N. Masoud, A. H. Nayfeh, and D. T. Mook, "Cargo pendulation reduction of ship-mounted cranes," Nonlinear Dynamics, vol. 35, no. 3, pp. 299-311, 2004.

[3] Z. N. Masoud, A. H. Nayfeh, and N. A. Nayfeh, "Sway reduction on quay-side container cranes using delayed feedback controller: simulations and experiments," Journal of Vibration and Control, vol. 11, no. 8, pp. 1103-1122, 2005.

[4] H. Schaub, "Rate-based ship-mounted crane payload pendulation control system," Control Engineering Practice, vol. 16, no. 1, pp. 132-145, 2008.

[5] S.-K. Cho and H.-H. Lee, "A fuzzy-logic antiswing controller for three-dimensional overhead cranes," ISA Transactions, vol. 41, no. 2, pp. 235-243, 2002.

[6] D. Liu, J. Yi, D. Zhao, and W. Wang, "Adaptive sliding mode fuzzy control for a two-dimensional overhead crane," Mechatronics, vol. 15, no. 5, pp. 505-522, 2005.

[7] H. M. Omar and A. H. Nayfeh, "Gantry cranes gain scheduling feedback control with friction compensation," Journal of Sound and Vibration, vol. 281, no. 1-2, pp. 1-20, 2005.

[8] W. Singhose, "Command shaping for flexible systems: a review of the first 50 years," International Journal of Precision Engineering and Manufacturing, vol. 10, no. 4, pp. 153-168, 2009.

[9] K. L. Sorensen, W. Singhose, and S. Dickerson, "A controller enabling precise positioning and sway reduction in bridge and gantry cranes," Control Engineering Practice, vol. 15, no. 7, pp. 825-837, 2007.

[10] J. Vaughan, A. Yano, and W. Singhose, "Comparison of robust input shapers," Journal of Sound and Vibration, vol. 315, no. 4-5, pp. 797-815, 2008.

[11] K. Terashima, Y. Shen, and K. Yano, "Modeling and optimal control of a rotary crane using the straight transfer transformation method," Control Engineering Practice, vol. 15, no. 9, pp. 1179-1192, 2007. 
[12] G. P. Starr, "Swing-free transport of suspended objects with a path-controlled robot manipulator," Journal of Dynamic Systems, Measurement and Control, vol. 107, no. 1, pp. 97-100, 1985.

[13] D. R. Strip, "Swing-free transport of suspended objects: a general treatment," IEEE Transactions on Robotics and Automation, vol. 5, no. 2, pp. 234-236, 1989.

[14] R. L. Kress, J. F. Jansen, and M. W. Noakes, "Experimental implementation of a robust damped-oscillation control algorithm on a full-sized, two-degree-of-freedom, AC induction motordriven crane," in Proceedings of the 5th International Symposium on Robotics and Manufacturing: Research, Education, and Applications (ISRAM '94), pp. 585-592, Maui, Hawaii, USA, 1994.

[15] G. G. Parker, K. Groom, J. Hurtado, R. D. Robinett, and F. Leban, "Command shaping boom crane control system with nonlinear inputs," in Proceedings of the IEEE International Conference on Control Applications (CCA '99), vol. 2, pp. 1774-1778, Kohala Coast, Hawaii, USA, August 1999.

[16] G. G. Parker, K. Groom, J. E. Hurtado, J. Feddema, R. D. Robinett, and F. Leban, "Experimental verification of a command shaping boom crane control system," in Proceedings of the American Control Conference (ACC '99), pp. 86-90, San Diego, Calif, USA, June 1999.

[17] K. A. Alhazza and Z. N. Masoud, "A novel wave-form command-shaping control with application on overhead cranes," in Proceedings of the ASME Dynamic Systems and Control Conference (DSCC '10), pp. 331-336, Cambridge, Mass, USA, September 2010, DSCC2010-4132.

[18] K. A. Alhazza, A. Al-Shehaima, and Z. N. Masoud, "A continuous modulated wave-form command-shaping for damped overhead cranes," in Proceedings of the ASME International Design Engineering Technical Conferences \& Computers and Information in Engineering Conference, Washington, DC, USA, August 2011, DETC2011-48336.

[19] K. A. Alhazza, "Experimental validation on a continuous modulated wafe-form command shaping applied on damped systems," in Topics in Dynamics of Civil Structures, Volume 4, Proceedings of the 31st IMAC Conference on Structural Dynamics, Proceedings of the Society for Experimental Mechanics Series 39, Springer, Garden Grove, Calif, USA, 2013.

[20] W. E. Singhose, L. J. Porter, and W. P. Seering, "Input shaped control of a planar Gantry crane with hoisting," in Proceedings of the JIn American Control Conference, pp. 97-100, Albuquerque, NM, USA, 1997.

[21] Z. N. Masoud and M. F. Daqaq, "A graphical approach to inputshaping control design for container cranes with hoist," IEEE Transactions on Control Systems Technology, vol. 14, no. 6, pp. 1070-1077, 2006.

[22] M. Kenison and W. Singhose, "Input shaper design for doublependulum planar gantry cranes," in Proceedings of the IEEE International Conference on Control Applications (CCA '99), pp. 539-544, Kohala Coast, Hawaii, USA, August 1999.

[23] D. Kim and W. Singhose, "Reduction of double-pendulum bridge crane oscillations," in Proceedings of the 8th International Conference on Motion and Vibration Control (MOVIC '06), pp. 300-305, Daejeon, Korea, 2006.

[24] Z. N. Masoud, K. A. Alhazza, M. A. Majeed, and E. A. Abu-Nada, "A hybrid command-shaping control system for highly accelerated double-pendulum gantry cranes," in Proceedings of the ASME International Design Engineering Technical Conferences (DETC '09) and Computers and Information in Engineering Conference (CIE '09), pp. 1809-1817, San Diego, Calif, USA, September 2009, Paper no. DETC2009-87501.
[25] Z. N. Masoud and K. A. Alhazza, "Command-shaping control system for double-pendulum gantry cranes," in Proceedings of the ASME International Design Engineering Technical Conferences \& Computers and Information in Engineering Conference, Washington, DC, USA, August 2011, Paper no. DETC201148400 .

[26] Y. Wang, F. Gao, and F. J. Doyle III, "Survey on iterative learning control, repetitive control, and run-to-run control," Journal of Process Control, vol. 19, no. 10, pp. 1589-1600, 2009.

[27] L. A. Scardua, J. J. da Cruz, and A. H. R. Costa, "Optimal control of ship unloaders using reinforcement learning," Advanced Engineering Informatics, vol. 16, no. 3, pp. 217-227, 2002.

[28] S. Arimoto, S. Kawamura, and F. Miyazaki, "Bettering operation of robots by learning," Journal of Robotic Systems, vol. 1, no. 2, pp. 123-140, 1984.

[29] D. A. Bristow and A. G. Alleyne, "A manufacturing system for microscale robotic deposition," in Proceedings of the American Control Conference, pp. 2620-2625, June 2003.

[30] H. Elci, R. W. Longman, M. Phan, J.-N. Juang, and R. Ugoletti, "Discrete frequency based learning control for precision motion control," in Proceedings of the IEEE International Conference on Systems, Man and Cybernetics, pp. 2767-2773, October 1994.

[31] W. Messner, R. Horowitz, W.-W. Kao, and M. Boals, "A new adaptive learning rule," IEEE Transactions on Automatic Control, vol. 36, no. 2, pp. 188-197, 1991.

[32] M. Norrlöf, "An adaptive iterative learning control algorithm with experiments on an industrial robot," IEEE Transactions on Robotics and Automation, vol. 18, no. 2, pp. 245-251, 2002.

[33] D. I. Kim and S. Kim, "An iterative learning control method with application for CNC machine tools," IEEE Transactions on Industry Applications, vol. 32, no. 1, pp. 66-72, 1996.

[34] D. de Roover and O. H. Bosgra, "Synthesis of robust multivariable iterative learning controllers with application to a wafer stage motion system," International Journal of Control, vol. 73, no. 10, pp. 968-979, 2000.

[35] H. Havlicsek and A. Alleyne, "Nonlinear control of an electrohydraulic injection molding machine via iterative adaptive learning," IEEE/ASME Transactions on Mechatronics, vol. 4, no. 3, pp. 312-323, 1999.

[36] F. Gao, Y. Yang, and C. Shao, "Robust iterative learning control with applications to injection molding process," Chemical Engineering Science, vol. 56, no. 24, pp. 7025-7034, 2001.

[37] M. Pandit and K.-H. Buchheit, "Optimizing iterative learning control of cyclic production processes with application to extruders," IEEE Transactions on Control Systems Technology, vol. 7, no. 3, pp. 382-390, 1999.

[38] S. S. Garimella and K. Srinivasan, "Application of iterative learning control to coil-to-coil control in rolling," in Proceedings of the American Control Conference, vol. 2, pp. 1230-1234, Alcoa Center, Pa, USA, June 1995.

[39] S. A. Saab, "A stochastic iterative learning control algorithm with application to an induction motor," International Journal of Control, vol. 77, no. 2, pp. 144-163, 2004.

[40] A. D. Barton, P. L. Lewin, and D. J. Brown, "Practical implementation of a real-time iterative learning position controller," International Journal of Control, vol. 73, no. 10, pp. 992-999, 2000.

[41] W. Hoffmann, K. Peterson, and A. G. Stefanopoulou, "Iterative learning control for soft landing of electromechanical valve actuator in camless engines," IEEE Transactions on Control Systems Technology, vol. 11, no. 2, pp. 174-184, 2003. 
[42] Y. Q. Chen and K. L. Moore, "A practical iterative learning pathfollowing control of an omni-directional vehicle," Asian Journal of Control, vol. 4, no. 1, pp. 90-98, 2002. 

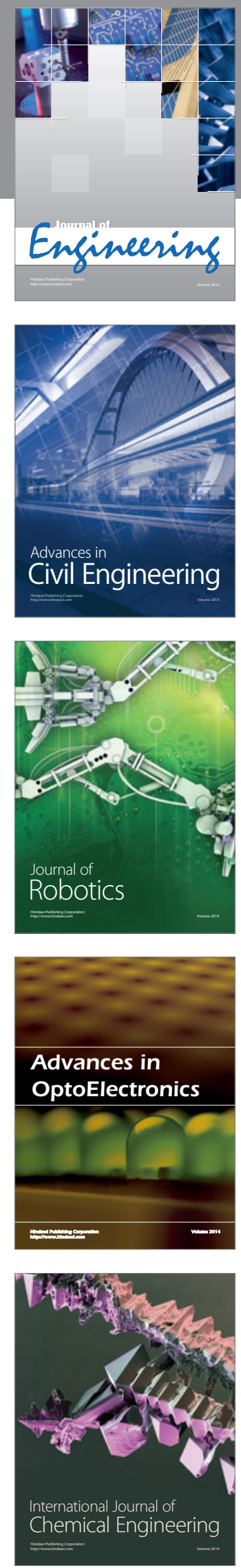

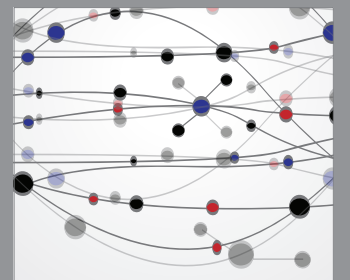

The Scientific World Journal
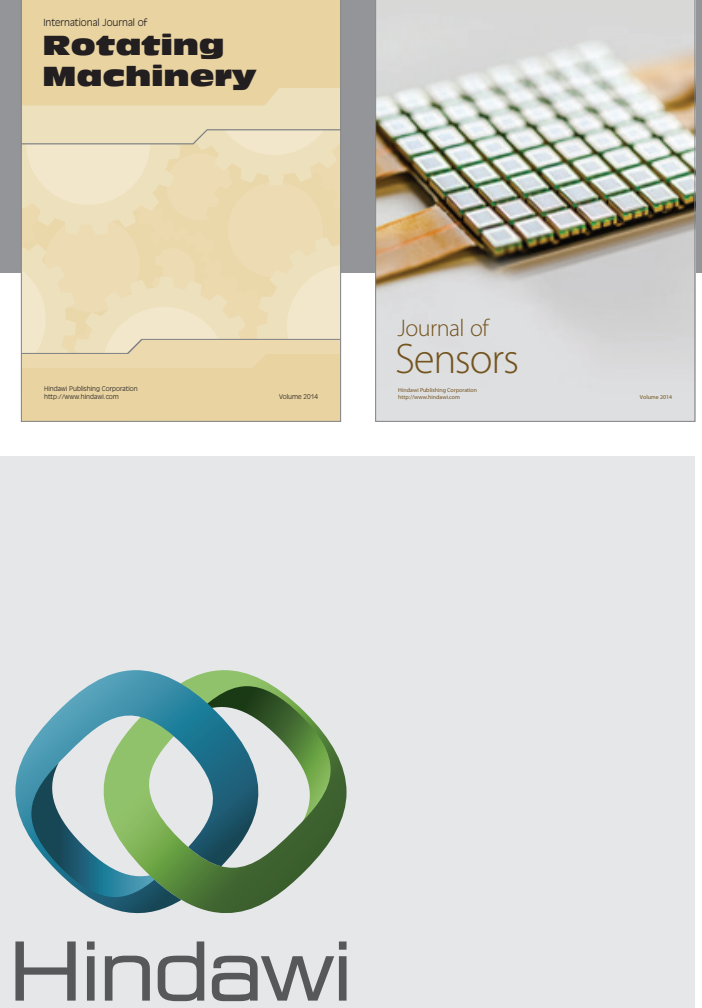

Submit your manuscripts at http://www.hindawi.com
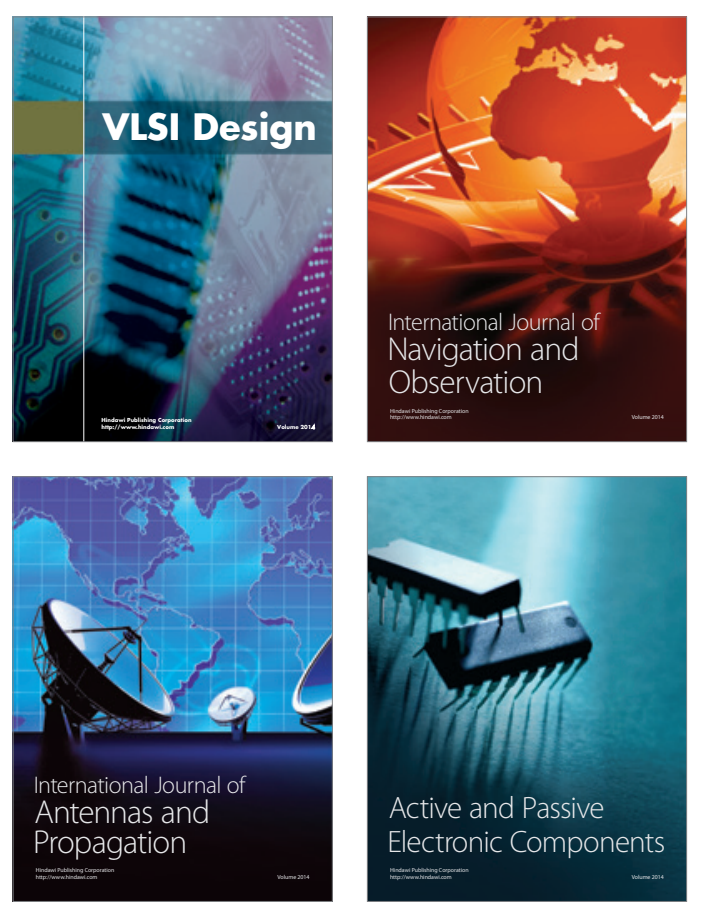
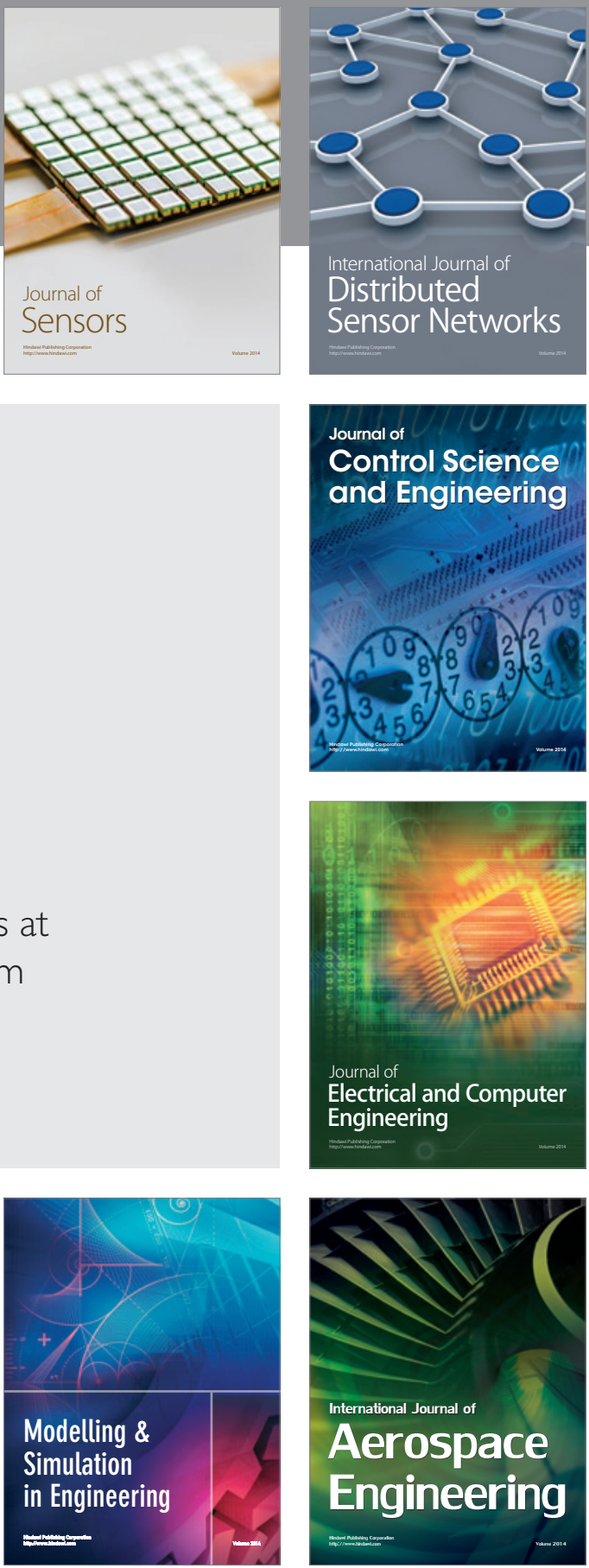

Journal of

Control Science

and Engineering
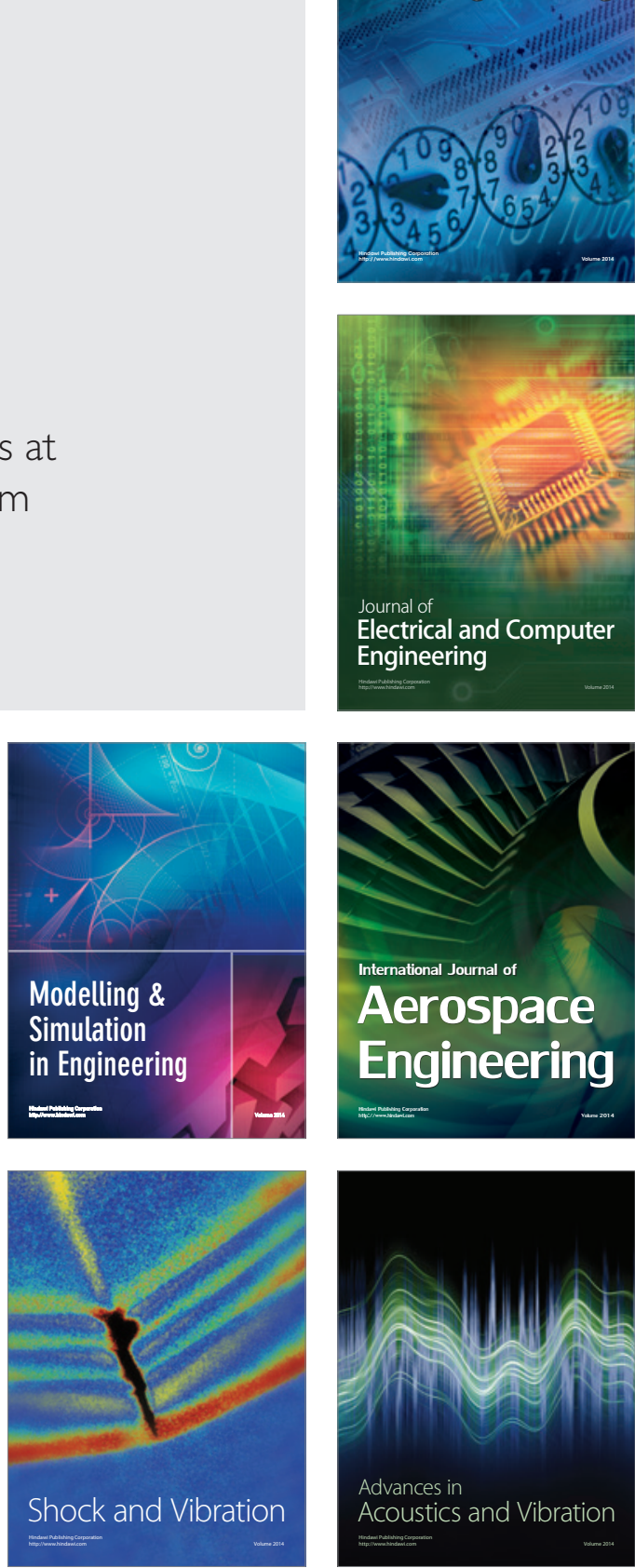